\title{
Preparation, characterization and in vitro evaluation of tablets containing microwave-assisted solid dispersions of apremilast
}

\author{
Jyotsana R. Madan ${ }^{1, A-F}$, Akshaya R. Pawar ${ }^{1, B-E}$, Rajesh B. Patil ${ }^{1, B-D, F}$, Rajendra Awasthi ${ }^{2, C-F}$, Kamal Dua ${ }^{3, C-F}$ \\ ${ }^{1}$ Department of Pharmaceutics, Smt. Kashibai Navale College of Pharmacy, Savitribai Phule Pune University, India \\ ${ }^{2}$ Amity Institute of Pharmacy, Amity University, Noida, India \\ ${ }^{3}$ Discipline of Pharmacy, Graduate School of Health, University of Technology, Sydney, Australia \\ A - research concept and design; $\mathrm{B}$ - collection and/or assembly of data; $\mathrm{C}$ - data analysis and interpretation; \\ $D$ - writing the article; $E$ - critical revision of the article; $F$ - final approval of the article
}

\section{Address for correspondence \\ Jyotsana R. Madan}

E-mail: jyotsna.madan@sinhgad.edu

\section{Funding sources \\ None declared}

Conflict of interest

None declared

Received on July 21, 2018

Reviewed on September 15, 2018

Accepted on November 15, 2018

Cite as

Madan JR, Pawar AR, Patil RB, Awasthi R, Dua K. Preparation, characterization and in vitro evaluation of tablets containing microwave-assisted solid dispersions of apremilast. Polim Med. 2018;48(1):17-24. doi:10.17219/pim/99801

DOI

$10.17219 / \mathrm{pim} / 99801$

Copyright

( 2018 by Wroclaw Medical University

This is an article distributed under the terms of the

Creative Commons Attribution Non-Commercial License

(http://creativecommons.org/licenses/by-nc-nd/4.0/)

\begin{abstract}
Background. Solid dispersions are among the techniques successfully employed to enhance the dissolution of poorly water-soluble drugs. Microwave (MW)-assisted evaporative crystallization has been used to prepare solid dispersions of drugs and polymers.

Objectives. The aim of the study was to investigate the solubility of apremilast (APM) in water by exploring the effect of MW-assisted solid dispersion technology.
\end{abstract}

Material and methods. In the present study, solid dispersions of APM, a poorly water-soluble drug, were prepared. The solid dispersions were prepared using the conventional method (CM) and the MW-based solvent evaporation technique. Microwave energy was used to enhance the solubility and dissolution rate of APM. The physical mixture and solid dispersions were characterized using Fourier-transform infrared spectroscopy (FTIR), X-ray powder diffraction (XRPD), differential scanning calorimetry (DSC), and scanning electron microscopy (SEM). Apremilast tablets containing MW-assisted solid dispersions were prepared by the direct compression technique and compared with the marketed formulation (Aprezo tablets).

Results. The results obtained confirmed the conversion of crystalline APM to an amorphous form. The XRPD pattern of the MW-assisted formulation at a 2:1 ratio suggests the amorphous structure of APM within the formulation. Based on solubility studies results, Syloid ${ }^{\circledR}$ 244FP was selected as the best carrier. The dissolution study results suggested that the APM tablet prepared using MW-assisted solid dispersions at a 2:1 carrier/drug ratio improved the APM dissolution rate compared to the marketed formulation.

Conclusions. Based on the results, it can be concluded that the MW-assisted solid dispersion technique may be an effective approach to enhancing the dissolution profile of other poorly water-soluble drugs.

Key words: microwave, solubility, apremilast, solid dispersions 


\section{Introduction}

A drug being developed into an oral formulation requires sufficient solubility in the dissolution medium in order to obtain optimal dissolution rates. The Biopharmaceutics Classification System (BCS) recognizes the importance of the rate of dissolution and aqueous solubility as key factors in the determination of the oral absorption of drugs.

Solid dispersions are among the techniques successfully employed to enhance the dissolution of poorly watersoluble drugs. ${ }^{1-9}$ Traditionally, solid dispersions are prepared by the melting, melting-solvent or solvent methods. Newer methods for the manufacture of solid dispersions include hot spin mixing, ${ }^{10}$ supercritical fluid technology and hot-melt extrusion (HME). ${ }^{11,12}$ Researchers are also investigating the potential of microwaves (MWs) to enhance solubility and bioavailability of poorly soluble drugs using the formation of solid dispersions and nanocomposite materials. In a recent study, MW-assisted evaporative crystallization was used to prepare solid dispersions of drugs and polymers. ${ }^{13}$

Microwaves are part of the electromagnetic spectrum, the frequency range of which is $0.3-300 \mathrm{GHz}$. The migration of MW within materials causes molecular oscillation, which leads to the generation of heat. ${ }^{14,15}$ The MW approach to heating is different from conventional heating, in which heat transfer takes place from the surface to the inner core. In the MW approach, heat is generated in the material and then passes to the entire volume, with a constant heating rate leading to uniform and deep heating of materials. ${ }^{14,16}$ Microwave technology is rapid, cost-effective, energy-saving, and environmentally safe. The crystalline drug is generally converted into an amorphous form, which means the approach can be used for the improvement of the dissolution profiles of BCS class II and IV drugs. ${ }^{14,17}$

Apremilast (APM) is a novel, orally available small molecule inhibitor of type 4 cyclic nucleotide phosphodiesterase (PDE4). It is indicated in the treatment of active psoriatic arthritis in adults and moderate to severe plaque psoriasis. ${ }^{18-21}$ It is practically insoluble in aqueous buffers, irrespective of $\mathrm{pH}$ range; it is soluble in acetone, acetonitrile, methyl ethyl ketone, methylene chloride, and tetrahydrofuran. The BCS classifies APM as having low solubility and low permeability (i.e., BCS class 4). Very poor solubility of APM in water influences the dissolution of the drug in aqueous media. Its oral bioavailability has been reported to be $20-33 \% .^{22,23}$ In order to achieve an acceptable dissolution profile, crystal forms of APM or reduction of the APM particle size have been studied to improve its dissolution. . $^{24} 25$

Considering the various attempts at improving solubility of APM with different approaches, the objective of present study was to increase the solubility of APM in water by exploring the effect of MW-assisted solid dispersion technology.

\section{Material and methods}

Apremilast was provided for the study by Glenmark Pharmaceuticals Ltd. (Nashik, India); APM tablets (Aprezo, the reference tablet) were purchased from the same company. Syloid $^{\circledR}$ 244FP and Syloid ${ }^{\circledR}$ XDP 3150 were purchased from Grace GmbH \& Co. KG (Worms, Germany). Polyethylene glycol (PEG) 6000, polyvinylpyrrolidone (PVP), $\beta$-cyclodextrin, lactose monohydrate, ethyl cellulose, and magnesium stearate were obtained from Research Lab Fine Chem Industries (Mumbai, India). Idacol Erythrosine Food Red-4 coloring agent was obtained from Roha Dyechem Pvt. Ltd. (Mumbai, India). All other reagents were of analytical grade.

\section{Determination of apremilast solubility in various buffers}

Saturation solubility studies of APM in water, $\mathrm{pH} 1.2$ acidic buffer, $\mathrm{pH} 2, \mathrm{pH} 4, \mathrm{pH} 6.8$, and $\mathrm{pH} 7.4$ phosphate buffers, as well as $\mathrm{pH} 6.8$ phosphate buffer with $0.15 \%$ sodium laureth sulfate (SLS) were conducted, using a magnetic stirrer (REMI Instruments Ltd., Mumbai, India). All media were prepared in individual flasks, and an excess amount of APM $(\approx 50 \mathrm{mg})$ was weighed and transferred into the flasks. The flasks were placed on a magnetic stirrer at a speed of $200 \mathrm{rpm}$ for $24 \mathrm{~h}$ at $37^{\circ} \mathrm{C}$. After $24 \mathrm{~h}$, the solution was centrifuged at $2000 \mathrm{rpm}$ for $15 \mathrm{~min}$. The supernatants were diluted with the respective media. Absorbance was measured at $230 \mathrm{~nm}$ using a Shimadzu V-630UV Visible Spectrophotometer (Shimadzu Corp., Kyoto, Japan) and solubility was calculated. ${ }^{6,26}$

\section{Preparation of physical mixtures of carriers and apremilast}

Apremilast and carriers (Syloid ${ }^{\circledR}$ 244FP (S244), Syloid ${ }^{\circledR}$ XDP 3150 (S3150), PEG 6000, PVP, and $\beta$-cyclodextrin) were each passed through a No. 100 sieve and physical mixtures (PMs) were prepared by mixing pre-weighed amounts of APM with each carrier at a 1:1 ratio.

\section{Preliminary trials for the preparation of solid dispersions by the conventional method}

We prepared solid dispersions of APM with the 5 selected carriers - S244, S3150, PEG 6000, PVP, and $\beta$-cyclodextrin - at a carrier/drug ratio of 1:1. The solid dispersions of APM with S244, S3150 and $\beta$-cyclodextrin were prepared using the solvent evaporation method: APM (250 mg) was dissolved in $3 \mathrm{~mL}$ of ethanol; after complete dissolution, the solution was transferred into a round-bottom flask with a polymer carrier. The solvent was evaporated at $45^{\circ} \mathrm{C}$ and dried in a Hei-VAP Value HB/G5 rotary evaporator (Heidolph Instruments $\mathrm{GmbH} \& \mathrm{CO}$. KG, Schwabach, Germany) for $2 \mathrm{~h}$, and the residues were passed through a No. 85 sieve. 
To prepare solid dispersions of APM in PEG 6000 and PVP, APM (250 mg) was dissolved in $3 \mathrm{~mL}$ of ethanol; after complete dissolution, the solutions were transferred to beakers holding the polymeric carriers PEG 6000 and PVP. The solvent was evaporated at $45^{\circ} \mathrm{C}$ and the resulting residues were dried in a hot air oven for $1 \mathrm{~h}$ and stored in a desiccator. Subsequently, the residues were ground in a mortar and passed through a No. 85 sieve.

On the basis of solubility studies, solid dispersions of APM with S244 at ratios of 2:1 and 3:1 were also prepared. All the solid dispersions were stored in vials at room temperature until further use. These batches were identified with the symbol CM. ${ }^{27,28}$

\section{The preparation of solid dispersions by the microwave-assisted solvent evaporation method}

For the microwaving process, we used a CATA 2R MW oven (Catalyst Systems, Pune, India), containing an inbuilt mode stirrer to ensure even MW distribution. Accurately weighed $0.5 \mathrm{~g}$ amounts of physically mixed S244 with APM at ratios of 1:1, 2:1 and 3:1 were dissolved in $3 \mathrm{~mL}$ of ethanol. After complete dissolution, the solutions were transferred to silica crucibles. The crucibles were kept in a MW oven at $560 \mathrm{~W}$ for $3 \mathrm{~min}$, until the solvent completely evaporated. ${ }^{13,29,30}$ The prepared solid dispersions were passed through a No. 85 sieve and stored in vials at room temperature until further use. These formulations were identified with the symbol MW.

\section{Characterization of the solid dispersions}

\section{Drug content}

The drug content of all the prepared solid dispersions was calculated. For each solid dispersion, an accurately weighed amount of solid dispersion containing $30 \mathrm{mg}$ of APM was transferred to a $100 \mathrm{~mL}$ volumetric flask, diluted to $100 \mathrm{~mL}$ with methanol and sonicated for $30 \mathrm{~min}$ for complete solubilization of the drug. The mixture was filtered using Whatman grade 41 filter paper and the absorbance was measured at $230 \mathrm{~nm}$. The drug content was calculated using a Shimadzu V-1800 UV spectrophotometer (Shimadzu Corp., Kyoto, Japan). ${ }^{14,25}$

\section{Phase solubility studies}

The phase solubility studies were performed according to the method described by Higuchi and Connors. ${ }^{31}$ Physical mixtures and solid dispersions containing different carrier/APM ratios were prepared and added to glass vials containing $10 \mathrm{~mL}$ each of different media. Each vial was shaken in a mechanical shaker for $12 \mathrm{~h}$ to obtain equilibrium solubility, and the solution was allowed to equilibrate for $24 \mathrm{~h}$. Each solution was further centrifuged at $2000 \mathrm{rpm}$ for $10 \mathrm{~min}$ in an ultra-centrifuge and filtered through Whatman grade 41 filter paper. An aliquot was suitably diluted with distilled water and analyzed using the Shimadzu V-1800 UV spectrophotometer at $230 \mathrm{~nm}^{32}$

\section{Fourier-transform infrared study}

The Fourier-transform infrared spectra (FTIR) of APM, the PM of APM with S244 and solid dispersion (Batch 7, MW 2:1) were recorded over a range of 4000-400 $\mathrm{cm}^{-1}$ to study the principal peaks with an Affinity-1 FTIR spectrophotometer (Shimadzu Corp.) using the potassiumbromide (KBr) disc method.

\section{X-ray powder diffraction analysis of apremilast}

The X-ray powder diffraction (XRPD) spectra of APM, S244, the PM of APM with S244 and all the solid dispersions were recorded using a Smart Lab high power powder X-ray diffractometer (Rigaku Corp., Tokyo, Japan) with $\mathrm{Cu}$ as a target filter, a voltage/current of $40 \mathrm{kV} / 40 \mathrm{~mA}$ and a scan speed of $4^{\circ} / \mathrm{min}$. The samples were analyzed at a $2 \theta$ angle range from $10^{\circ}$ to $89^{\circ}$. The step time was $0.5 \mathrm{~s}$ and the acquisition time was 1 h. ${ }^{33,34}$

\section{Differential scanning calorimetry analysis}

Thermograms of APM, S244, the PM of APM with S244 and all the solid dispersions were recorded by using a Shimadzu DSC-60 differential scanning calorimeter (Shimadzu Corp.). An empty aluminum pan was used as a reference. The differential scanning calorimetry (DSC) measurements were performed at a heating rate of $10^{\circ} \mathrm{C} / \mathrm{min}$ from $30^{\circ} \mathrm{C}$ to $300^{\circ} \mathrm{C} .^{29,35}$

\section{Surface morphology}

The surface morphology of APM, the PM of APM with S244 and solid dispersion (Batch 7, MW 2:1) was studied using a Supra 5 scanning electron microscope (SEM) (Carl Zeiss AG, Oberkochen, Germany) with an accelerating voltage of $10 \mathrm{kV}$.

\section{Preparation of apremilast tablets using the direct compression technique}

All the ingredients as shown in Table 1 were individually passed through a No. 60 sieve. A solid dispersion containing $30 \mathrm{mg}$ of APM and microcrystalline cellulose were mixed to obtain a uniform mixture. The other ingredients were weighed, mixed and tablets were pressed using an $11 \mathrm{~mm}$ concave round punch on a Rimek compression machine (Karnavati Engineering Ltd., Ahmedabad, India). The post-compression parameters of the uncoated tablets were evaluated before they were coated. ${ }^{36}$ 
Table 1. Composition of apremilast (APM) tablets prepared by direct compression method

\begin{tabular}{|l|c|}
\multicolumn{1}{|c}{ Ingredients } & mg/Tablet \\
\hline S244 + APM MW (2:1) & 90.00 \\
Lactose monohydrate & 140.00 \\
Croscarmellose sodium & 16.30 \\
Microcrystalline cellulose & 41.50 \\
Magnesium stearate & 2.20 \\
Total weight & 290.00 \\
\hline
\end{tabular}

\section{Preparation of coating solution for film coating}

Ethyl cellulose $(1 \% \mathrm{w} / \mathrm{w})$ was weighed accurately and added to approx. $5 \mathrm{~mL}$ of acetone in a beaker with continuous stirring using the handshake method. Idacol Erythrosine Food Red-4 coloring agent (Roha Dyechem Pvt. Ltd., Mumbai, India) was then added. The stirring was continued for $15 \mathrm{~min}$.

\section{Film coating of tablets}

The compressed tablets were coated in a conventional coating pan (Avon Engineering Work, Mumbai, India) with an inlet air temperature of $50-55^{\circ} \mathrm{C}$, a tablet bed temperature of $35-40^{\circ} \mathrm{C}$ and exhaust air temperature of $35-42^{\circ} \mathrm{C}$. The pan speed, spray air pressure and solution spray rate were $25-30 \mathrm{rpm}, 3 \mathrm{~kg} / \mathrm{cm}^{2}$ and $20 \mathrm{~mL} / \mathrm{min}$, respectively. The tablets were dried for $15 \mathrm{~min}$ at $45^{\circ} \mathrm{C}$. The coating increased weight of the core tablets by $4 \%$.

\section{Dissolution studies}

The dissolution rates of the APM tablets and Aprezo (the reference tablet) were studied using a USP XXIII Dissolution Test Apparatus (Labindia Instruments, Mumbai, India) at $50 \mathrm{rpm}$ in $900 \mathrm{~mL}$ of phosphate buffer ( $\mathrm{pH}$ 6.8) with $0.15 \%$ SLS. The temperature of the dissolution medium was maintained at $37 \pm 0.5^{\circ} \mathrm{C}$. Aliquots $(5 \mathrm{~mL})$ were withdrawn at 5-, 10-, 20-, 30-, 40-, 50-, and 60-minute intervals and filtered. The absorbance of the filtered sample solution was measured using the Shimadzu V-1800 UV spectrophotometer at $230 \mathrm{~nm}$, and concentration of APM was determined from the standard calibration curve.

\section{Results and discussion}

\section{Solubility studies}

Our study showed that APM has poor solubility in all buffers. The highest solubility $(24.74 \pm 0.857 \mu \mathrm{g} / \mathrm{mL})$ was observed in phosphate buffer ( $\mathrm{pH}$ 6.8) with $0.15 \%$ SLS (Table 2). The FDA has also suggested phosphate buffer (pH 6.8) with $0.15 \%$ SLS as a dissolution medium for APM tablets. ${ }^{37}$
Table 2 . Results of solubility study of apremilast (APM) in different media

\begin{tabular}{|l|r|}
\multicolumn{1}{c|}{ Dissolution media } & $\begin{array}{r}\text { Solubility } \\
{[\mu \mathrm{g} / \mathrm{mL}]}\end{array}$ \\
Water & $5.4 \pm 0.298$ \\
$\mathrm{pH}$ 1.2, acidic buffer & $5.2 \pm 0.118$ \\
$\mathrm{pH}$ 2, phosphate buffer & $9.96 \pm 0.827$ \\
$\mathrm{pH}$ 4, phosphate buffer & $14.96 \pm 1.065$ \\
$\mathrm{pH}$ 6.8, phosphate buffer & $16.22 \pm 0.640$ \\
$\mathrm{pH}$ 7.4, phosphate buffer with 0.15\% SLS & $24.74 \pm 0.857$ \\
\hline
\end{tabular}

Data presents mean \pm standard deviation (SD), $n=3$.

All the carriers used in the preliminary trials showed an increase in the solubility of APM in both PMs and solid dispersions (Tables 3 and 4). Several studies on solid dispersions have been published, confirming the advantageous properties of solid dispersions in increasing the solubility and dissolution rate of poorly water-soluble drugs. Solid dispersions tend to reduce particle size, possibly to a molecular level, and change the crystalline state of drugs, thereby promoting their solubility. ${ }^{38,39}$ The results of phase solubility studies indicate that the solubility of APM was maximally enhanced when S244 was used as the carrier for preparing solid dispersions utilizing the conventional method (CM). This is because the increase in surface area, along with the potential existence of the drug in pores in an amorphous (rather than crystalline) form, can aid in enhancing solubility. When MW heating is used for solvent evaporation, a $3 \mathrm{~mL}$ solution completely evaporates within $3 \mathrm{~min}$. Thus, the time consumed by MW-assisted solvent evaporation was significantly shorter than in the conventional method.

Further, when a solid dispersion is prepared using the MW-assisted solvent evaporation method, it is possible that APM molecules are transported into the pores by capillary action and stored in the pores in a partially crystalline and partially amorphous form. Earlier findings suggest that particle size decreased to the submicron range when using the MW-assisted technique, thus improving dissolution rate. ${ }^{13}$ However, at a low carrier/drug ratio (1:1), surface adsorption of APM was noted. These results were also supported by the DSC thermogram. When the carrier/drug ratio is increased to 2:1, APM is more confined to the pores and can exist in an amorphous or a semi-crystalline state.

Table 3. Results of phase solubility studies of physical mixtures (PMs)

\begin{tabular}{|l|c|}
\multicolumn{1}{|c|}{ Physical mixtures } & $\begin{array}{c}\text { Solubility } \\
{[\mu \mathrm{g} / \mathrm{mL}]}\end{array}$ \\
\hline S244 + APM (1:1) & $28 \pm 0.221$ \\
Syloid XDP 3150 + APM (1:1) & $22 \pm 0.123$ \\
PVP + APM (1:1) & $18 \pm 0.344$ \\
PEG 6000 + APM (1:1) & $19 \pm 0.243$ \\
B-cyclodextrin + APM (1:1) & $20 \pm 0.354$ \\
\hline
\end{tabular}

Data presents mean \pm standard deviation $(S D), n=3$. 
Table 4. Results of drug content and solubility studies of solid dispersion

\begin{tabular}{|c|c|c|c|}
\hline Batch code & Solid dispersion & $\begin{array}{c}\text { Drug content } \\
{[\%]}\end{array}$ & $\begin{array}{l}\text { Solubility } \\
{[\mu \mathrm{g} / \mathrm{mL}]}\end{array}$ \\
\hline APM 1 & S244 + APM (CM 1:1) & $97.24 \pm 3.24$ & $28 \pm 0.154$ \\
\hline APM 2 & Syloid XDP 3150 + APM (CM 1:1) & $96.88 \pm 2.84$ & $23 \pm 0.231$ \\
\hline APM 3 & PVP+ APM (CM 1:1) & $99.12 \pm 5.31$ & $24 \pm 0.269$ \\
\hline APM 4 & PEG 6000 + APM (CM 1:1) & $97.37 \pm 2.57$ & $23 \pm 0.347$ \\
\hline APM 5 & $\beta$-cyclodextrin + APM (CM 1:1) & $96.56 \pm 3.62$ & $21 \pm 0.215$ \\
\hline APM 6 & S244 + APM (MW 1:1) & $98.23 \pm 3.64$ & $30 \pm 0.364$ \\
\hline APM 7 & S244 + APM (MW 2:1) & $100.32 \pm 2.81$ & $33 \pm 0.385$ \\
\hline APM 8 & S244 + APM (MW 3:1) & $98.41 \pm 4.11$ & $33 \pm 0.316$ \\
\hline
\end{tabular}

Data presents mean \pm standard deviation (SD), $\mathrm{n}=3$

A similar reduction in crystallinity was observed as the carrier/drug ratio is increased to 3:1.

On the basis of our solubility studies, S244 was selected as the best carrier for the further studies.

\section{Fourier-transform infrared study}

The FTIR spectrum of APM (Fig. 1) shows the characteristic strong $\mathrm{N}-\mathrm{H}$ stretching peak for APM at $3364 \mathrm{~cm}^{-1}$. The FTIR spectrum of APM also shows the characteristic peak at $1764 \mathrm{~cm}^{-1}$ due to amide carbonyl $(\mathrm{C}=\mathrm{O})$, along with the peaks between $2837 \mathrm{~cm}^{-1}$ and $3081 \mathrm{~cm}^{-1}$ for aliphatic and aromatic benzene ring $\mathrm{C}-\mathrm{H}$ stretching. The peak for amide $\mathrm{N}-\mathrm{H}$ bending was observed at $1597 \mathrm{~cm}^{-1}$ and the peak for $\mathrm{C}-\mathrm{O}$ stretching was observed at $1164 \mathrm{~cm}^{-1}$. The FTIR spectrum of S244 showed a stronger intensity band from $900 \mathrm{~cm}^{-1}$ to $1300 \mathrm{~cm}^{-1}$ due to $\mathrm{Si}-\mathrm{O}$ stretching of the silanol group. The FTIR spectra for the PM of APM with S244 and for solid dispersion (Batch 7, MW 2:1) show the combined individual characteristic peaks for APM and S244. The individual peaks for APM are more intense in the PM as compared to the solid dispersion. This suggests that in the PM, the physical interaction of APM with S244

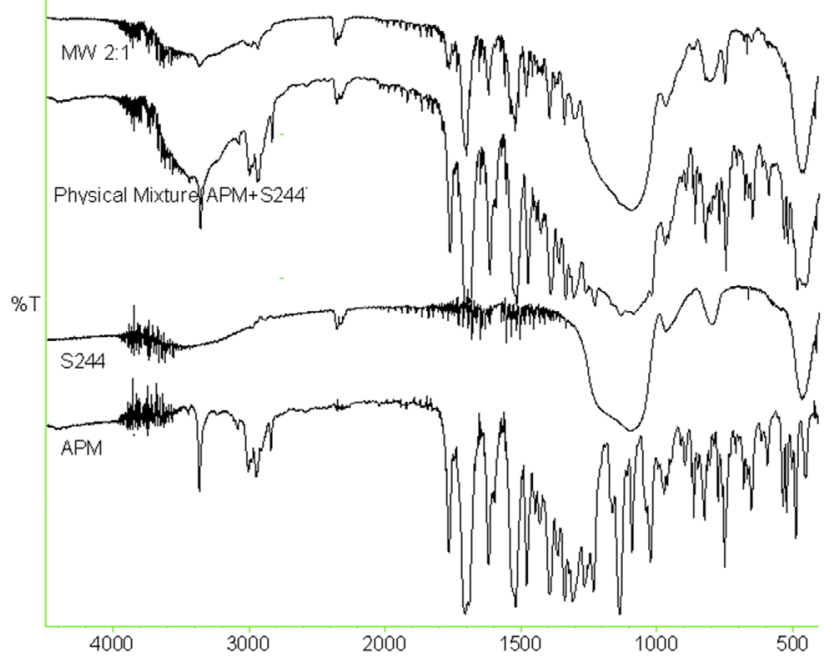

Fig. 1. Fourier transform infrared (FTIR) spectra of solid dispersion (MW 2:1), the physical mixture (PM) of S244 and apremilast (APM), S244 alone and APM alone is weak; possibly APM is only physically interacting with the outer surface of the silica particles. In the solid dispersion, the individual peaks for APM are weak, which suggests that MW irradiation results in pronounced physical interaction between APM at the deeper sites of the S244 pores. The FTIR spectrum also clearly suggests that in both the PM and the solid dispersion, APM was physically adsorbed rather than undergoing any chemical interaction on the surface of the porous silica particles of S244.

\section{Differential scanning calorimetry and X-ray diffraction}

Differential scanning calorimetry and XRPD were employed to investigate the crystal lattice of pure APM and APM solid dispersions with S244. Figure 2 depicts the DSC thermograms for S244, a PM of APM and S244, solid dispersions of S244 and APM obtained using the CM and the MW-assisted solvent evaporation method. Apremilast was characterized by a single sharp melting endothermic peak at $157.56{ }^{\circ} \mathrm{C}$. The peak onset temperature and heat of fusion $(\Delta \mathrm{Hf})$ were $155.01^{\circ} \mathrm{C}$ and $111.0595 \mathrm{Jg}^{-1}$, respectively. This characteristic peak appeared in the PMs and in the CM batches at carrier/drug ratios of both 1:1 and 2:1, with slight variations in terms of the melting peak depression and broadening, indicating the transition from

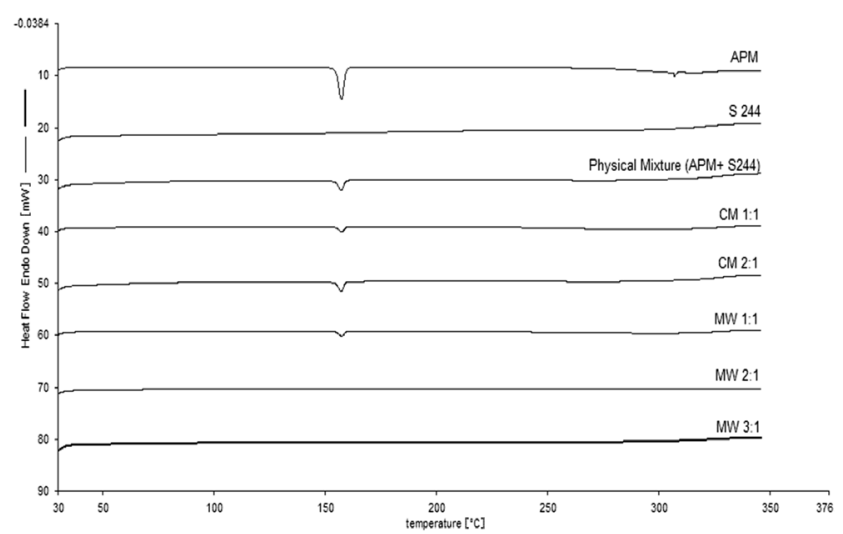

Fig. 2. Differential scanning calorimetry (DSC) thermograms of apremilast (APM), S244, the physical mixture (PM) of S244 and APM, solid dispersion (CM 1:1, CM 2:1), solid dispersion (MW 1:1, MW 2:1, and MW 3:1) 
a crystalline to a semi-crystalline state. In the MW solid dispersion of APM with S244 at a carrier/drug ratio of 1:1, a melting peak of less intensity was detected. At this ratio, the pore volume of S244 was insufficient for hosting extra APM molecules, and the residual APM instead remained on the external surface of S244. However, the melting peak was completely absent in the 2:1 and 3:1 MW solid dispersions, confirming the amorphous state of APM within these formulations.

Figure 3 displays the XRPD patterns of APM, S244 and the MW solid dispersions with ratios of $1: 1$ and $2: 1$. The characteristic diffraction peaks observed at $10.09^{\circ}, 11.87^{\circ}$, $13.53^{\circ}, 16.34^{\circ}, 26.09^{\circ}$, and $26.92^{\circ}$ correspond to the powder diffraction pattern for pure APM, while the absence of diffraction peaks in the solid dispersions confirms their amorphous structure. The crystalline state of APM in the physical mixture of S244 and APM is evident from the characteristic diffraction peaks. The less intense diffraction peaks in the MW solid dispersion at a ratio of 1:1 indicates the partially crystalline state of APM deposited between the pore walls as a result of the blockage of pores with viscous molten APM. However, the XRPD pattern in the MW solid dispersion at a ratio of 2:1 suggests the amorphous structure of APM within the formulation.

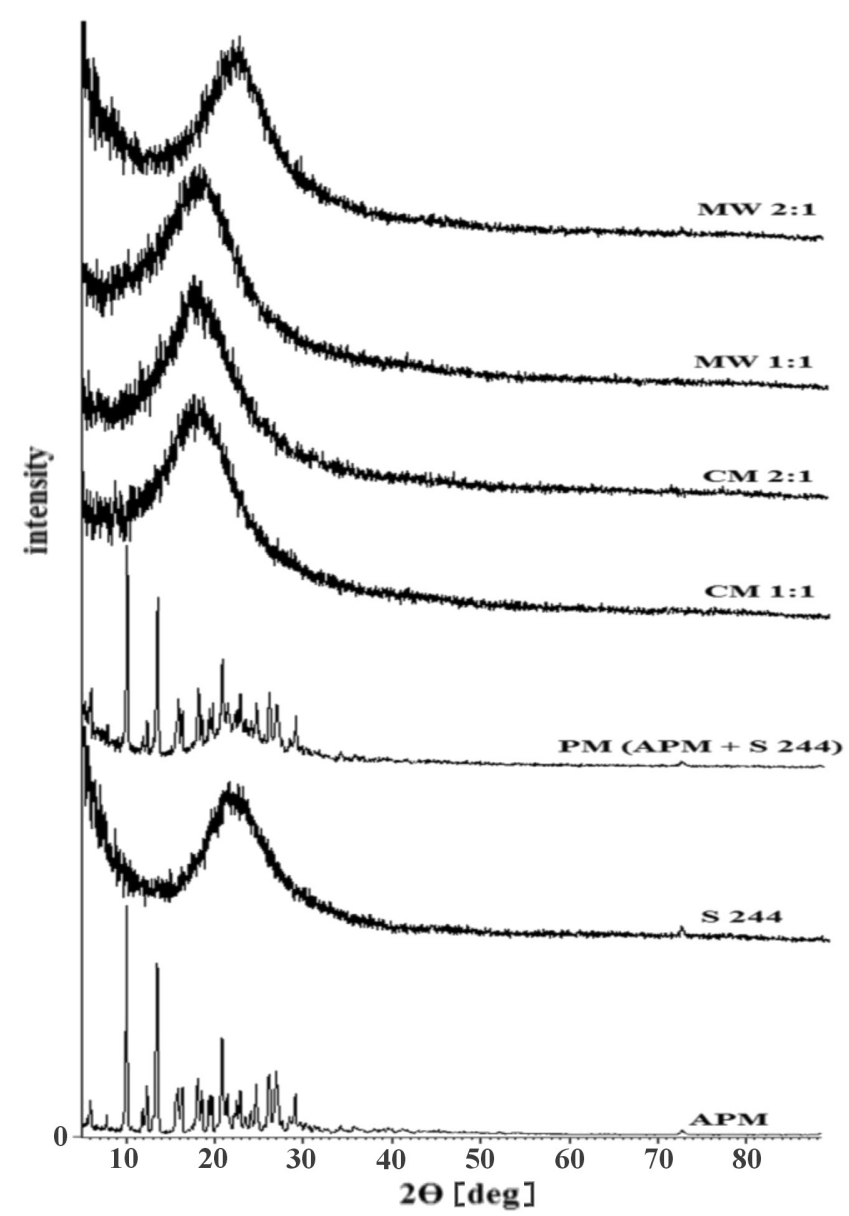

Fig. 3. X-ray powder diffraction (XRPD) patterns of solid dispersion (MW 2:1, MW 1:1), solid dispersion (CM 1:1), the physical mixture (PM) of S244and apremilast (APM), S244, and APM
It also confirms that the APM was confined within the pores of S244. These XRPD results are compatible with the DSC observations discussed above. S224 is a non-ordered porous silicon dioxide with a neutral $\mathrm{pH}$ and has randomly oriented pores with an average pore diameter of $19 \mathrm{~nm} .{ }^{40}$ The single-crystal structure of APM has been reported for its ethanol hemisolvate solvomorph. ${ }^{24}$ This single-crystal structure is most stable experimentally determined conformation of APM, and this conformation was therefore used to measure the size and shape of APM. Another reason for using the single-crystal structure is that very small changes in the conformation of a molecule or changes in the hydrogen bonds around the molecule can have major effects on its size, shape, surface area and volume.

As shown in Fig. 4, the most widely spaced atoms in APM are 14.7 $\AA$ apart. The unit cell dimensions required to fit the conformer of APM are $1.29 \times 1.29 \times 1.47 \mathrm{~nm}$, calculated using Discovery Studio (Biovia, San Diego, USA) with the PyMOL interface (Schrödinger LLC, New York, USA). This suggests that the size of APM is below $1.9 \mathrm{~nm}$, whereas the pore size of S244 is in the range of 19-20 nm. Therefore, the restricted pore size of S244 may have inhibited the crystallization of APM inside the pores. In summary, the transformation of APM from crystalline to amorphous form is highly dependent on the selection of an optimum carrier/drug ratio and on the formulation method.

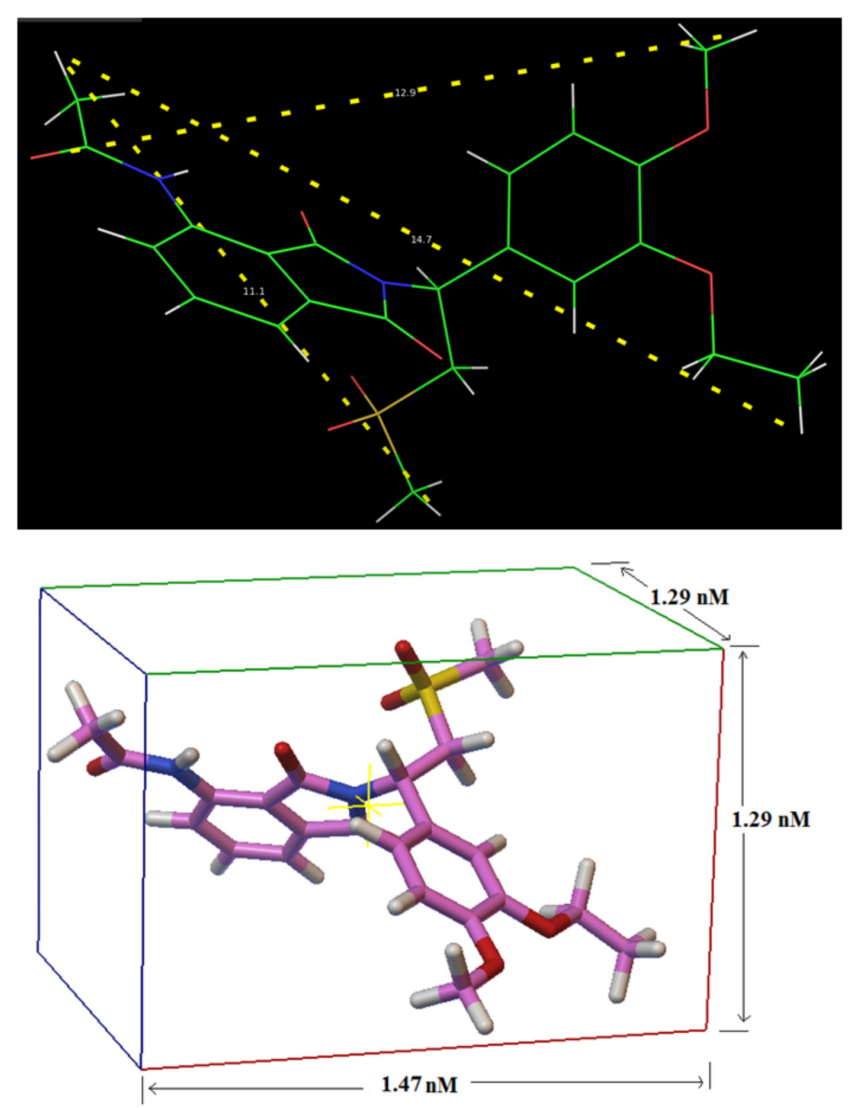

Fig. 4. Representation of a 3-dimensional apremilast (APM) molecule with inter-atomic distances shown 


\section{Surface morphology}

Scanning electron microscopy images of APM, the physical mixture of APM and S244 and the MW solid dispersion of S244 and APM (2:1) are presented in Fig. 5. The SEM images of pure APM showed irregularly shaped crystals of the drug with large particles, showing its existence in the crystalline form. When APM was converted into solid dispersions, the morphology changed to an amorphous state with a fine particle size.

\section{Preparation of apremilast tablets}

Based on the results of our solubility studies, DSC thermograms and X-ray powder diffraction patterns, the best solid dispersion was found to be Batch 7 (MW $2: 1)$. This was used to prepare tablets. The post-compression parameters of the uncoated tablets are presented in Table 5.

\section{Dissolution studies}

From the dissolution profiles, it is evident that the APM tablets prepared with the MW-assisted solid dispersion at a 2:1 ratio had a significantly better APM dissolution rate as compared to the marketed formulation (Aprezo tablets) (Fig. 6). Factors such as the lack of a crystalline form, the increased surface area of the drug, and the hydrophilic surface of S244 helped improve the dissolution of APM. When this formulation comes into contact with the dissolution media, a rapid release of APM in the form of fine particles occurs, possibly because of desorption of APM by the influx of the dissolution media inside the pores of S244. ${ }^{28}$ These results are in agreement with the findings of earlier researchers, ${ }^{39}$ who used MW-induced solid dispersions to improve the solubility of APM.

\section{Conclusions}

In summary, solid dispersions of APM, a poorly water-soluble drug, with Syloid 244FP as a carrier were prepared by CM and MW-assisted method. The latter was found to be a suitable and less time-consuming method. The prepared solid dispersions were evaluated for physicochemical parameters. The study shows that the dissolution rate of APM can be improved using Syloid 244FP as a carrier. The transformation of APM from crystalline to amorphous form is highly dependent on the selection of an optimum carrier/ drug ratio and formulation method. The tablet formulation of solid dispersion (Batch 7, MW 2:1) (S244 with APM, MW 2:1) showed a higher dissolution rate when compared with the reference tablets.
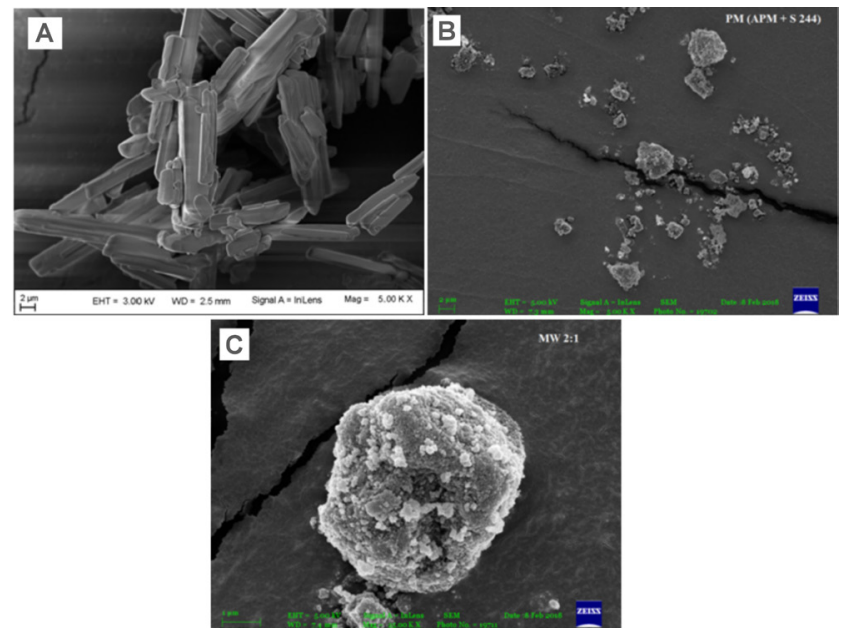

Fig. 5. A - scanning electron micrographs of pure apremilast (APM) $B$ - the physical mixture (PM) of S244 and APM; C - solid dispersion (batch 7, MW 2:1). Scales are given on the individual micrographs

Table 5. Results of uncoated Apremilast (APM) tablet

\begin{tabular}{|c|c|c|c|}
\hline $\begin{array}{c}\text { Average weight } \\
{[\mathrm{mg}]}\end{array}$ & $\begin{array}{c}\text { Friability } \\
{[\%]}\end{array}$ & $\begin{array}{c}\text { Disintegration time } \\
{[\mathrm{s}]}\end{array}$ & $\begin{array}{c}\text { Hardness } \\
{\left[\mathrm{kg} / \mathrm{cm}^{2}\right]}\end{array}$ \\
\hline 290 & $0.63 \pm 0.05$ & $66 \pm 1.52$ & $7.5 \pm 0.5$ \\
\hline
\end{tabular}

Data are presented as mean \pm standard deviation (SD), $\mathrm{n}=3$.

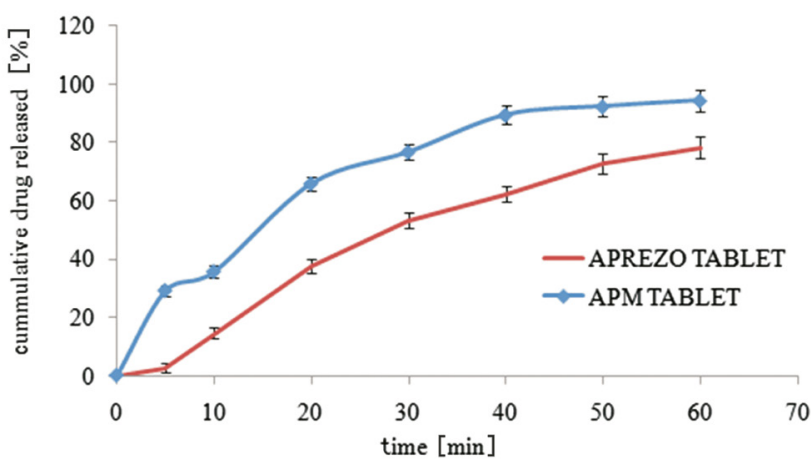

Fig. 6. Comparative dissolution profiles of prepared apremilast (APM) tablets and the marketed formulation (Aprezo tablets). Data are presented as means \pm standard deviation (SD), $\mathrm{n}=3$

\section{References}

1. Serajuddin A. Solid dispersion of poorly water-soluble drugs: Early promises, subsequent problems, and recent breakthroughs. J Pharm Sci. 1999;88(10):1058-1066.

2. Chiou WL, Riegelman S. Pharmaceutical applications of solid dispersion systems. J Pharm Sci. 1971;60(9):1281-1302.

3. Dua K, Pabreja K, Ramana MV, Bukhari NI. Preparation, characterization, and in vitro evaluation of aceclofenac PVP-solid dispersions. J Disper Sci Technol. 2011;32(8):1151-1157.

4. Schver GCRM, Lee PI. Combined effects of supersaturation rates and doses on the kinetic-solubility profiles of amorphous solid dispersions based on water-insoluble poly (2-hydroxyethyl methacrylate) hydrogels. Mol Pharm. 2018;15(5):2017-2026. doi:10.1021/acs. molpharmaceut.8b00162

5. Muhammad RS, Bashir S, Mahmood A, et al. Application of various polymers and polymers based techniques used to improve solubility of poorly water soluble drugs: A review. Acta Poloniae Pharmaceutica. 2017;74(2):347-356. 
6. Madan JR, Pawar KT, Dua K. Solubility enhancement studies on lurasidone hydrochloride using mixed hydrotropy. Int J Pharm Investig. 2015;5(2):114-120.

7. Dua K, Pabreja K, Ramana MV, Lather V. Dissolution behavior of $\beta$-cyclodextrin molecular inclusion complexes of aceclofenac. J Pharm Bioallied Sci. 2011;3(3):417-425.

8. Dua K, Ramana MV, Sara UV, et al. Investigation of enhancement of solubility of norfloxacin beta-cyclodextrin in presence of acidic solubilizing additives. Curr Drug Deliv. 2007;4(1):21-25.

9. Dua K, Pabreja K, Gorajana A. Dissolution behaviour of aceclofenac-PVP coprecipitates. Ars Pharm. 2012;53(3):7-12.

10. Dittgen $M$, Fricke $S$, Gerecke $H$, Osterwald $H$. Hot spin mixing: A new technology to manufacture solid dispersions. I: Testosterone. Pharmazie. 1995;50(3):225-226.

11. Van Nijlen T, Brennan K, Van der Mooter G, Blaton N, Kinget R, Augustijns P. Improvement of the dissolution rate of artemisinin by means of supercritical fluid technology and solid dispersion. Int J Pharm. 2003;254(2):173-181.

12. Ghaderi R, Artursson P, Carlfors J. Preparation of biodegradable microparticles using solution enhanced dispersion by supercritical fluids (SEDS). J Pharm Res. 1999;16(5):676-681.

13. Radacsi N, Stefanidis G, Szabo-Revesz P, Ambrus R. Analysis of niflumic acid prepared by rapid microwave-assisted evaporation. J Pharm Biomed Anal. 2014;98:16-21.

14. Alshehri S, Shakeel F, Ibrahim M, et al. Influence of the microwave technology on solid dispersions of mefenamic acid and flufenamic acid. PLOS ONE. 2017;12(7):e0182011.

15. Wang W, Zhao C, Sun J, et al. Quantitative measurement of energy utilization efficiency and study of influence factors in typical microwave heating process. Energy. 2015;87:678-685.

16. Xiqiang Z, Wenlong W, Hongzhen L, Yampeng M, Chunyuan M, Zhanlong $S$. Temperature rise and weight loss characteristics of wheat straw under microwave heating. J Anal Appl Pyrol. 2014;107:59-66.

17. Moneghini M, Bellich B, Baxa P, Princivalle F. Microwave generated solid dispersion containing ibuprofen. Int J Pharm. 2008;361(1-2):125-130.

18. Martin BC, Thomas LW, Dann FJ. Apremilast for the treatment of psoriatic arthritis. Dermatol Online J. 2017;23(2):1-12.

19. Manhart R, Rich PC. Nail psoriasis. Clin Exp Rheumatol. 2015;33(5 Suppl 93):S7-S13.

20. Papp K, Cather JC, Rosoph L, et al. Efficacy of apremilast in the treatment of moderate to sever psoriasis: A randomized controlled trial. Lancet. 2012;380(9843):738-746.

21. Oram Y, Akkaya AD. Treatment of nail psoriasis: Common concepts and new trends. Dermatol Res Pract. 2013;2013:180496. doi: $10.1155 / 2013 / 180496$

22. EMA (2014). Full prescribing information available at: http://www.ema. europa.eu/docs/enGB/documentlibrary/EPAR_Public_assesmentreport/human/003746/WC500182629.pdf. Accessed January 21, 2018.

23. FDA (2014a). Full prescribing information available at: http://www. acceessdata.fda.gov/drugsatfda_docs/nda/2014/205437 Origl s000 ChemR.pdf. Accessed November 11, 2017.

24. Wu YD, Zhang XL, Liu XH, et al. The preparation, characterization, structure and dissolution analysis of apremilast solvatomorphs. Acta Crystallogr C: Struct Chem. 2017;73(Pt 4):305-313.

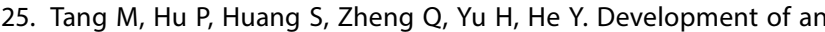
extended-release formulation for apremilast and a level $A$ in vitro-in vivo correlation study in beagle dogs. Chem Pharm Bull. 2016;64(11):1607-1615.

26. Madan JR, Pawar KT, Dua K. Solubility enhancement studies on lurasidone hydrochloride using mixed hydrotropy. Int J Pharm Investig. 2015:5(2):114-120.

27. Madan JR, Kamate VJ, Dua K, Awasthi R. Improving the solubility of nevirapine using a hydrotropy and mixed hydrotropy based solid dispersion approach. Polim Med. 2017;47(2):83-90.

28. Lai J, Lin W, Scholes P, Li M. Investigating the effects of loading factors on the in vitro pharmaceutical performance of mesoporous material as drug carrier ibuprofen. Materials (Basel). 2017;10(2):E150. doi:103390/ma10020150

29. Gorajana A, Rajendra A, Yew LM, Dua K. Preparation and characterization of cefuroxime axetil solid dispersion using hydrophilic carriers. Int J Pharm Investig. 2015;5(3):171.
30. Talib $\mathrm{H}$. The application of microwave formulation and isothermal titration calorimetry for pharmaceutical compounds [PhD thesis]. University of Huddersfield; 2014.

31. Higuchi TK, Connors KA. Phase solubility techniques. Adv Anal Chem Instrum. 1965;4:117-212.

32. Maheshwari RK, Indurkhya A. Formulation and evaluation of aceclofenac injection made by mixed hydrotropic solubilization technique. Iran J Pharm Res. 2010;9(3):233-242.

33. Bhole PG, Patil VR. Enhancement of water solubility of felodipine by preparing solid dispersion using poly-ethylene glycol 6000 and polyvinyl alcohol. Asian J Pharm. 2014;28(3):240-244.

34. Gorajana A, Kit WW, Dua K. Characterization and solubility study of norfloxacin-polyethylene glycol, polyvinylpyrrolidone and carbopol 974P solid dispersions. Recent Pat Drug Deliv Formul. 2015;9(2):167-182.

35. Lyn LY, Sze HW, Rajendran A, Adinarayana G, Dua K, Garg S. Crystal modifications and dissolution rate of piroxicam. Acta Pharm. 2011;61(4):391-402.

36. Madan JR, Kamate VJ, Awasthi R, Dua K. Formulation, characterization and in-vitro evaluation of fast dissolving tablets containing gliclazide hydrotropic solid dispersions. Recent Pat Drug Deliv Formul. 2017;11(2):147-154.

37. FDA (2014b), Full prescribing information available at: https://www. accessdata.fda.gov/scrips/cder/ dissolution/dsp_getllData.cfm. Accessed July 24, 2017.

38. Vasconcelos T, Costa P. Development of a rapid dissolving ibuprofen solid dispersion. Pharm Res. 2007;16:676-681.

39. Maurya D, Belgamwar V, Tekade A. Microwave induced solubility enhancement of poorly water soluble atrovastatin calcium. J Pharm Pharmacol. 2010;62(11):1599-1606.

40. Lai J, Lin W, Scholes P, Li M. Investigating the effects of loading factors on the in vitro pharmaceutical performance of mesoporous materials as drug carriers for ibuprofen. Materials. 2017;10(2):150. doi:10.3390/ma10020150 